\title{
Szász-Baskakov type operators based on $q$-integers
}

Purshottam N Agrawal ${ }^{1}$, Harun Karsli ${ }^{2}$ and Meenu Goyal ${ }^{1 *}$

\section{"Correspondence:}

meenu.goyaliitr@yahoo.com

'Department of Mathematics,

Indian Institute of Technology

Roorkee, Roorkee, 247667, India

Full list of author information is

available at the end of the article

\begin{abstract}
In the present paper, we introduce the $q$-analog of the Stancu variant of Szász-Baskakov operators. We establish the moments of the operators by using the $q$-derivatives and then prove the basic convergence theorem. Next, the Voronovskaja type theorem and some direct results for the above operators are discussed. Also, the rate of convergence and weighted approximation by these operators in terms of modulus of continuity are studied. Then we obtain a point-wise estimate using the Lipschitz type maximal function. Lastly, we study the A-statistical convergence of these operators and also, in order to obtain a better approximation, we study a King type modification of the above operators.
\end{abstract}

MSC: 41A25; 26A15; 40A35

Keywords: Szász-Baskakov operators; rate of convergence; modulus of continuity; weighted approximation; point-wise estimates; Lipschitz type maximal function; statistical convergence

\section{Introduction}

In recent years, there has been intensive research on the approximation of functions by positive linear operators introduced by making use of $q$-calculus. Lupas [1] and Phillips [2] pioneered the study in this direction by introducing $q$-analogs of the well-known Bernstein polynomials. Derriennic [3] discussed modified Bernstein polynomials with Jacobi weights. Gupta [4] and Gupta and Heping [5] proposed the $q$-analogs of usual and discretely defined Durrmeyer operators and studied their approximation properties. In [6], Stancu introduced the positive linear operators $P_{n}^{(\alpha, \beta)}: C[0,1] \rightarrow C[0,1]$ by modifying the Bernstein polynomials as

$$
P_{n}^{(\alpha, \beta)}(f ; x)=\sum_{k=0}^{n} p_{n, k}(x) f\left(\frac{k+\alpha}{n+\beta}\right)
$$

where $p_{n, k}(x)=\left(\begin{array}{l}n \\ k\end{array}\right) x^{k}(1-x)^{n-k}, x \in[0,1]$ is the Bernstein basis function and $\alpha, \beta$ are any two real numbers satisfying $0 \leq \alpha \leq \beta$. Recently, Buyukyazici [7] introduced the Stancu type generalization of certain $q$-Baskakov operators and studied some local direct results for these operators. Subsequently, several authors (cf. [8-11] etc.) have considered such a modification for some other sequences of positive linear operators.

(2014 Agrawal et al.; licensee Springer. This is an Open Access article distributed under the terms of the Creative Commons Attribution License (http://creativecommons.org/licenses/by/2.0), which permits unrestricted use, distribution, and reproduction in any medium, provided the original work is properly cited. 
To approximate Lebesgue integrable functions defined on $[0, \infty)$, Gupta et al. [12] introduced the following positive linear operators:

$$
M_{n}(f ; x)=\sum_{v=0}^{\infty} q_{n, v}(x) \int_{0}^{\infty} b_{n, v}(t) f(t) d t
$$

where $b_{n, v}(x)=\frac{1}{B(v+1, n)} \frac{x^{v}}{(1+x)^{n+v+1}}, q_{n, v}(x)=e^{-n x} \frac{(n x)^{v}}{v !}, x \in[0, \infty)$, and studied the asymptotic approximation and error estimation in simultaneous approximation. Subsequently, for $f \in C_{\gamma}[0, \infty):=\left\{f \in C[0, \infty):|f(t)| \leq M_{f}\left(1+t^{\gamma}\right)\right.$ for some $\left.M_{f}>0, \gamma>0\right\}$, Gupta [13] introduced the following operators:

$$
S_{n}(f ; x)=\sum_{\nu=1}^{\infty} q_{n, v}(x) \int_{0}^{\infty} b_{n, v-1}(t) f(t) d t+e^{-n x} f(0)
$$

by considering the value of the function at zero explicitly, and studied an estimate of error in terms of the higher order modulus of continuity in simultaneous approximation for a linear combination of the operators (1.2), introduced by May [14]. Later on, Gupta and Noor [15] discussed some direct results in a simultaneous approximation for the operators (1.2).

In [16], Aral introduced Szász-Mirakjan operators based on $q$-integers and established some direct results and gave two representations of the $r$ th $q$-derivative of these operators. Aral and Gupta [17] studied the basic convergence theorem for the $r$ th order $q$-derivatives, a Voronovskaja type theorem, and some more properties of these operators. Agratini and Doğru [18] constructed Szász-King type operators and investigated the statistical convergence and the rate of local and global convergence for functions with a polynomial growth. Örkcü and Doğru [19] proposed two different modifications of $q$-Szász-Mirakjan Kantorovich operators and obtained the rate of convergence in terms of the modulus of continuity. In [20], they introduced Kantorovich type generalization of $q$-Szász-Mirakjan operators and discussed their $A$-statistical approximation properties.

Let the space $C_{\gamma}[0, \infty)$ be endowed with the norm $\|f\|_{\gamma}=\sup _{t \in[0, \infty)} \frac{|f(x)|}{\left(1+t^{\gamma}\right)}$ then for $f \in$ $C_{\gamma}[0, \infty), 0<q<1,0 \leq \alpha \leq \beta$ and each positive integer $n$, we introduce the following Stancu type modification of the operators (1.2) based on $q$-integers:

$$
\begin{aligned}
B_{n, q}^{(\alpha, \beta)}(f ; x)= & \sum_{\nu=1}^{\infty} q_{n, v}(q, x) q^{\nu-1} \int_{0}^{\infty / A} b_{n, v-1}(q, t) f\left(\frac{q^{v}[n]_{q} t+\alpha}{[n]_{q}+\beta}\right) d_{q} t \\
& +e_{q}\left(-[n]_{q} x\right) f\left(\frac{\alpha}{[n]_{q}+\beta}\right)
\end{aligned}
$$

where $q_{n, v}(q, x)=\frac{e_{q}\left(-[n]_{q} x\right)[n]_{q}^{v} x^{v}}{[v]_{q} !}$ and $b_{n, v}(q, t)=\frac{t^{v} q^{v(v-1) / 2}}{(1+t)^{n+v+1} B_{q}(v+1, n)}$.

For $\alpha=\beta=0$, we denote $B_{n, q}^{(\alpha, \beta)}(f ; x)$ by $B_{n, q}(f ; x)$.

Clearly, if $q \rightarrow 1^{-}$and $\alpha=\beta=0$, the operators defined by (1.3) reduce to the operators given by (1.2).

The purpose of the present paper is to study the basic convergence theorem, Voronovskaja type asymptotic formula, local approximation, rate of convergence, weighted approximation, point-wise estimation and $A$-statistical convergence of the operators (1.3). Fur- 
ther, to obtain a better approximation we also propose a modification of these operators by using a King type approach.

We recall that the $q$-analog of beta function of second kind [21,22] is defined by

$$
B_{q}(t, s)=K(A, t) \int_{0}^{\infty / A} \frac{x^{t-1}}{(1+x)_{q}^{t+s}} d_{q} x
$$

where $K(x, t)=\frac{1}{x+1} x^{t}\left(1+\frac{1}{x}\right)_{q}^{t}(1+x)_{q}^{1-t}$, and $(a+b)_{q}^{s}=\prod_{i=0}^{s-1}\left(a+q^{i} b\right), s \in \mathbb{Z}^{+}$.

In particular, for any positive integers $n, m$, we have

$$
K(x, n)=q^{\frac{n(n-1)}{2}}, \quad K(x, 0)=1
$$

and

$$
B_{q}(m, n)=\frac{\Gamma_{q}(m) \Gamma_{q}(n)}{\Gamma_{q}(m+n)} .
$$

\section{Moment estimates}

Lemma 1 For $B_{n, q}\left(t^{m} ; x\right), m=0,1,2$, one has

(1) $B_{n, q}(1 ; x)=1$;

(2) $B_{n, q}(t ; x)=\frac{[n]_{q} x}{[n-1]_{q}}$, for $n>1$;

(3) $B_{n, q}\left(t^{2} ; x\right)=\frac{q[n]_{q}^{2}}{[n-1]_{q}[n-2]_{q}} x^{2}+\frac{[2]_{q}[n]_{q}}{q[n-1]_{q}[n-2]_{q}} x$, for $n>2$.

Proof We observe that $B_{n, q}$ are well defined for the functions $1, t, t^{2}$. Thus, for every $x \in$ $[0, \infty)$, using (1.4) and (1.5), we obtain

$$
\begin{aligned}
B_{n, q}(1 ; x) & =\sum_{\nu=1}^{\infty} q_{n, v}(q, x) q^{\nu-1} \int_{0}^{\infty / A} b_{n, v-1}(q, t) d_{q} t+e_{q}\left(-[n]_{q} x\right) \\
& =\sum_{\nu=0}^{\infty} q_{n, v}(q, x)=1 .
\end{aligned}
$$

Next, for $f(t)=t$, again applying (1.4) and (1.5), we get

$$
\begin{aligned}
B_{n, q}(t ; x) & =\sum_{\nu=1}^{\infty} q_{n, v}(q, x) q^{\nu-1} \int_{0}^{\infty / A} b_{n, v-1}(q, t) q^{v} t d_{q} t \\
& =\frac{[n]_{q}}{[n-1]_{q}} x .
\end{aligned}
$$

Proceeding similarly, we have

$$
B_{n, q}\left(t^{2} ; x\right)=\frac{[2]_{q}[n]_{q}}{q[n-1]_{q}[n-2]_{q}} x+\frac{q[n]_{q}^{2}}{[n-1]_{q}[n-2]_{q}} x^{2}
$$

by using $[v+2]_{q}=[2]_{q}+q^{2}[v]_{q}$.

Lemma 2 For the operators $B_{n, q}^{(\alpha, \beta)}(f ; x)$ as defined in (1.3), the following equalities hold:

(1) $B_{n, q}^{(\alpha, \beta)}(1 ; x)=1$; 
(2) $B_{n, q}^{(\alpha, \beta)}(t ; x)=\frac{[n]_{q}^{2}}{\left([n]_{q}+\beta\right)[n-1]_{q}} x+\frac{\alpha}{[n]_{q}+\beta}$, for $n>1$;

(3) $B_{n, q}^{(\alpha, \beta)}\left(t^{2} ; x\right)=\left(\frac{[n]_{q}}{[n]_{q}+\beta}\right)^{2}\left\{\frac{\left(q[n]_{q}^{2}+2 \alpha[n-2]_{q}\right)}{[n-1]_{q}[n-2]_{q}} x^{2}+\frac{[2]_{q}[n]_{q}}{q[n-1]_{q}[n-2]_{q}} x\right\}+\left(\frac{\alpha}{[n]_{q}+\beta}\right)^{2}$, for $n>2$.

Proof This lemma is an immediate consequence of Lemma 1. Hence the details of its proof are omitted.

Lemma 3 For $f \in C_{B}[0, \infty)$ (the space of all bounded and uniform continuous functions on $[0, \infty)$ endowed with norm $\|f\|=\sup \{|f(x)|: x \in[0, \infty)\})$, one has

$$
\left\|B_{n, q}^{(\alpha, \beta)}(f ; x)\right\| \leq\|f\|
$$

Proof In view of (1.3) and Lemma 2, the proof of this lemma easily follows.

Remark 1 For every $q \in(0,1)$, we have

$$
B_{n, q}^{(\alpha, \beta)}((t-x) ; x)=\frac{\left([n]_{q}^{2}-\left([n]_{q}+\beta\right)[n-1]_{q}\right) x+\alpha[n-1]_{q}}{\left([n]_{q}+\beta\right)[n-1]_{q}}, \quad n>1,
$$

and

$$
\begin{aligned}
B_{n, q}^{(\alpha, \beta)}\left((t-x)^{2} ; x\right) & \\
= & \left\{1+\frac{q[n]_{q}^{4}}{\left([n]_{q}+\beta\right)^{2}[n-1]_{q}[n-2]_{q}}+(\alpha-1) \frac{2[n]_{q}^{2}}{\left([n]_{q}+\beta\right)^{2}[n-1]_{q}}\right\} x^{2} \\
& +\left\{\frac{[2]_{q}[n]_{q}^{3}}{q\left([n]_{q}+\beta\right)^{2}[n-1]_{q}[n-2]_{q}}-\frac{2 \alpha}{[n]_{q}+\beta}\right\} x+\frac{\alpha^{2}}{\left([n]_{q}+\beta\right)^{2}}, \quad n>2 \\
= & : \gamma_{n, q}(x),
\end{aligned}
$$

say.

\section{Main results}

Theorem 1 Let $0<q_{n}<1$ and $A>0$. Then for each $f \in C_{\gamma}[0, \infty)$, the sequence $\left\{B_{n, q_{n}}^{(\alpha, \beta)}(f ; x)\right\}$ converges to $f$ uniformly on $[0, A]$ if and only if $\lim _{n \rightarrow \infty} q_{n}=1$.

Proof First, we assume that $\lim _{n \rightarrow \infty} q_{n}=1$.

We have to show that $\left\{B_{n, q_{n}}^{(\alpha, \beta)}(f ; x)\right\}$ converges to $f$ uniformly on $[0, A]$.

From Lemma 2, we see that

$$
B_{n, q_{n}}^{(\alpha, \beta)}(1 ; x) \rightarrow 1, \quad B_{n, q_{n}}^{(\alpha, \beta)}(t ; x) \rightarrow x, \quad B_{n, q_{n}}^{(\alpha, \beta)}\left(t^{2} ; x\right) \rightarrow x^{2},
$$

uniformly on $[0, A]$ as $n \rightarrow \infty$.

Therefore, the well-known property of the Korovkin theorem implies that $\left\{B_{n, q_{n}}^{(\alpha, \beta)}(f ; x)\right\}$ converges to $f$ uniformly on $[0, A]$ provided $f \in C_{\gamma}[0, \infty)$.

We show the converse part by contradiction. Assume that $q_{n}$ does not converge to 1 . Then it must contain a subsequence $\left\{q_{n_{k}}\right\}$ such that $q_{n_{k}} \in(0,1), q_{n_{k}} \rightarrow a \in[0,1)$ as $k \rightarrow \infty$. 
Thus, $\frac{1}{\left[n_{k}+s\right]_{q_{n_{k}}}}=\frac{1-q_{n_{k}}}{1-\left(q_{n_{k}}\right)^{n_{k}+s}} \rightarrow(1-a)$ as $k \rightarrow \infty$. Choosing $n=n_{k}, q=q_{n_{k}}$ in $B_{n, q_{n}}^{(\alpha, \beta)}\left(t^{2} ; x\right)$, from Lemma 2, we have

$$
B_{n, q_{n}}^{(\alpha, \beta)}\left(t^{2} ; x\right) \rightarrow \frac{(a+2 \alpha(1-a)) x^{2}}{(1+(1-a) \beta)^{2}}+\frac{\left(1-a^{2}\right) x}{a(1+(1-a) \beta)^{2}}+\frac{(1-a)^{2} \alpha^{2}}{(1+(1-a) \beta)^{2}} \neq x^{2} \quad \text { as } k \rightarrow \infty
$$

which leads us to a contradiction. Hence, $\lim _{n \rightarrow \infty} q_{n}=1$. This completes the proof.

Theorem 2 (Voronovskaja type theorem) Let $f \in C_{\gamma}[0, \infty)$ and $q_{n} \in(0,1)$ be a sequence such that $q_{n} \rightarrow 1$ and $q_{n}^{n} \rightarrow 0$ as $n \rightarrow \infty$. Suppose that $f^{\prime \prime}(x)$ exists at a point $x \in[0, \infty)$, then we have

$$
\lim _{n \rightarrow \infty}[n]_{q_{n}}\left(B_{n, q_{n}}^{(\alpha, \beta)}(f ; x)-f(x)\right)=(\alpha-\beta x) f^{\prime}(x)+(1-\alpha) x(1-x) f^{\prime \prime}(x) .
$$

Proof By Taylor's formula we have

$$
f(t)=f(x)+(t-x) f^{\prime}(x)+\frac{1}{2} f^{\prime \prime}(x)(t-x)^{2}+r(t, x)(t-x)^{2}
$$

where $r(t, x)$ is the Peano form of the remainder and $\lim _{t \rightarrow x} r(t, x)=0$.

Applying $B_{n, q}^{(\alpha, \beta)}(f, x)$ to the both sides of (3.1), we have

$$
\begin{aligned}
{[n]_{q_{n}}\left(B_{n, q_{n}}^{(\alpha, \beta)}(f ; x)-f(x)\right)=} & {[n]_{q_{n}} f^{\prime}(x) B_{n, q_{n}}^{(\alpha, \beta)}((t-x) ; x)+\frac{1}{2}[n]_{q_{n}} f^{\prime \prime}(x) B_{n, q_{n}}^{(\alpha, \beta)}\left((t-x)^{2} ; x\right) } \\
& +[n]_{q_{n}} B_{n, q}^{(\alpha, \beta)}\left((t-x)^{2} r(t, x) ; x\right) .
\end{aligned}
$$

In view of Remark 1 , we have

$$
\lim _{n \rightarrow \infty}[n]_{q_{n}} B_{n, q_{n}}^{(\alpha, \beta)}((t-x) ; x)=\alpha-\beta x
$$

and

$$
\lim _{n \rightarrow \infty}[n]_{q_{n}} B_{n, q_{n}}^{(\alpha, \beta)}\left((t-x)^{2} ; x\right)=2 x(1-x)(1-\alpha) .
$$

Now, we shall show that

$$
[n]_{q_{n}} B_{n, q_{n}}^{(\alpha, \beta)}\left(r(t, x)(t-x)^{2} ; x\right) \rightarrow 0
$$

when $n \rightarrow \infty$.

By using the Cauchy-Schwarz inequality, we have

$$
B_{n, q_{n}}^{(\alpha, \beta)}\left(r(t, x)(t-x)^{2} ; x\right) \leq \sqrt{B_{n, q_{n}}^{(\alpha, \beta)}\left(r^{2}(t, x) ; x\right)} \sqrt{B_{n, q_{n}}^{(\alpha, \beta)}\left((t-x)^{4} ; x\right)} .
$$

We observe that $r^{2}(x, x)=0$ and $r^{2}(\cdot, x) \in C_{\gamma}[0, \infty)$. Then it follows from Theorem 1 that

$$
\lim _{n \rightarrow \infty} B_{n, q_{n}}^{(\alpha, \beta)}\left(r^{2}(t, x) ; x\right)=r^{2}(x, x)=0,
$$


in view of the fact that $B_{n, q_{n}}^{(\alpha, \beta)}\left((t-x)^{4} ; x\right)=O\left(\frac{1}{[n]_{q_{n}}^{2}}\right)$. Now, from (3.4) and (3.5), we get

$$
\lim _{n \rightarrow \infty} B_{n, q_{n}}^{(\alpha, \beta)}\left(r(t, x)(t-x)^{2} ; x\right)=0
$$

and from (3.2), (3.3), and (3.6), we get the required result.

Theorem 3 (Voronovskaja type theorem) Let $f \in C_{\gamma}[0, \infty)$ and $q_{n} \in(0,1)$ be a sequence such that $q_{n} \rightarrow 1$ and $q_{n}^{n} \rightarrow 0$ as $n \rightarrow \infty$. If $f^{\prime \prime}(x)$ exists on $[0, \infty)$, then

$$
\lim _{n \rightarrow \infty}[n]_{q_{n}}\left(B_{n, q_{n}}^{(\alpha, \beta)}(f ; x)-f(x)\right)=(\alpha-\beta x) f^{\prime}(x)+(1-\alpha) x(1-x) f^{\prime \prime}(x)
$$

holds uniformly on $[0, A]$, where $A>0$.

Proof Let $x \in[0, A]$. The remainder part of the proof of this theorem is similar to that of the proof of the previous theorem. So we omit it.

\subsection{Local approximation}

For $C_{B}[0, \infty)$, let us consider the following $K$-functional:

$$
K_{2}(f, \delta)=\inf _{g \in W^{2}}\left\{\|f-g\|+\delta\left\|g^{\prime \prime}\right\|\right\}
$$

where $\delta>0$ and $W^{2}=\left\{g \in C_{B}[0, \infty): g^{\prime \prime} \in C_{B}[0, \infty)\right\}$. By [23] there exists an absolute constant $C>0$ such that

$$
K_{2}(f, \delta) \leq C \omega_{2}(f, \sqrt{\delta})
$$

where

$$
\omega_{2}(f, \sqrt{\delta})=\sup _{0<h \leq \sqrt{\delta}} \sup _{x \in[0, \infty)}|f(x+2 h)-2 f(x+h)+f(x)|
$$

is the second order modulus of smoothness of $f$. By

$$
\omega(f, \delta)=\sup _{0<h \leq \delta} \sup _{x \in[0, \infty)}|f(x+h)-f(x)|
$$

we denote the usual modulus of continuity of $f \in C_{B}[0, \infty)$.

Theorem 4 Let $f \in C_{B}[0, \infty)$ and $q \in(0,1)$. Then, for every $x \in[0, \infty)$ and $n \geq 2$, we have

$$
\left|B_{n, q}^{(\alpha, \beta)}(f(t) ; x)-f(x)\right| \leq C \omega_{2}\left(f, \delta_{n}(x)\right)+\omega\left(f, \frac{\left([n]_{q} q^{n-1}-\beta[n-1]_{q}\right) x+\alpha[n-1]_{q}}{\left([n]_{q}+\beta\right)[n-1]_{q}}\right)
$$

where $C$ is an absolute constant and

$$
\delta_{n}(x)=\left(B_{n, q}^{(\alpha, \beta)}\left((t-x)^{2} ; x\right)+\left[\frac{\left([n]_{q} q^{n-1}-\beta[n-1]_{q}\right) x+\alpha[n-1]_{q}}{\left([n]_{q}+\beta\right)[n-1]_{q}}\right]^{2}\right)^{1 / 2} .
$$


Proof For $x \in[0, \infty)$, we consider the auxiliary operators $\bar{B}_{n, q}^{(\alpha, \beta)}$ defined by

$$
\bar{B}_{n, q}^{(\alpha, \beta)}(f ; x)=B_{n, q}^{(\alpha, \beta)}(f ; x)-f\left(\frac{[n]_{q}^{2} x}{\left([n]_{q}+\beta\right)[n-1]_{q}}+\frac{\alpha}{[n]_{q}+\beta}\right)+f(x) .
$$

From Lemma 2, we observe that the operators $\bar{B}_{n, q}^{(\alpha, \beta)}$ are linear and reproduce the linear functions.

Hence

$$
\bar{B}_{n, q}^{(\alpha, \beta)}((t-x) ; x)=0
$$

Let $g \in W^{2}$. By Taylor's theorem, we have

$$
g(t)=g(x)+g^{\prime}(x)(t-x)+\int_{x}^{t}(t-u) g^{\prime \prime}(u) d u, \quad t \in[0, \infty) .
$$

Applying $\bar{B}_{n, q}^{(\alpha, \beta)}$ to the both sides of the above equation and using (3.11), we have

$$
\bar{B}_{n, q}^{(\alpha, \beta)}(g ; x)=g(x)+\bar{B}_{n, q}^{(\alpha, \beta)}\left(\int_{x}^{t}(t-u) g^{\prime \prime}(u) d u ; x\right) .
$$

Thus, by (3.10) we get

$$
\begin{aligned}
\left|\bar{B}_{n, q}^{(\alpha, \beta)}(g ; x)-g(x)\right| \\
\leq B_{n, q}^{(\alpha, \beta)}\left(\int_{x}^{t}|t-u|\left|g^{\prime \prime}(u)\right| d u ; x\right) \\
\quad+\int_{x} \frac{[n]_{q}^{2} x}{([n] q)[n-1]_{q}}+\frac{\alpha}{[n]_{q}+\beta}\left|\frac{[n]_{q}^{2} x}{\left([n]_{q}+\beta\right)[n-1]_{q}}+\frac{\alpha}{[n]_{q}+\beta}-u\right|\left|g^{\prime \prime}(u)\right| d u \\
\leq\left[B_{n, q}^{(\alpha, \beta)}\left((t-x)^{2} ; x\right)+\left(\frac{[n]_{q}^{2} x}{\left([n]_{q}+\beta\right)[n-1]_{q}}+\frac{\alpha}{[n]_{q}+\beta}-x\right)^{2}\right]\left\|g^{\prime \prime}\right\| \\
\leq \delta_{n}^{2}(x)\left\|g^{\prime \prime}\right\| .
\end{aligned}
$$

On other hand, by (3.10) and Lemma 3, we have

$$
\left|\bar{B}_{n, q}^{(\alpha, \beta)}(f ; x)\right| \leq\left|B_{n, q}^{(\alpha, \beta)}(f ; x)\right|+2\|f\| \leq 3\|f\| .
$$

Using (3.12) and (3.13) in (3.10), we obtain

$$
\begin{aligned}
\left|B_{n, q}^{(\alpha, \beta)}(f ; x)-f(x)\right| \leq & \left|\bar{B}_{n, q}^{(\alpha, \beta)}(f-g ; x)\right|+|(f-g)(x)|+\left|\bar{B}_{n, q}^{(\alpha, \beta)}(g ; x)-(g)(x)\right| \\
& +\left|f\left(\frac{[n]_{q}^{2} x}{\left([n]_{q}+\beta\right)[n-1]_{q}}+\frac{\alpha}{[n]_{q}+\beta}\right)-f(x)\right| \\
\leq & 4\|f-g\|+\delta_{n}^{2}(x)\left\|g^{\prime \prime}\right\|+\left|f\left(\frac{[n]_{q}^{2} x+\alpha[n-1]_{q}}{\left([n]_{q}+\beta\right)[n-1]_{q}}\right)-f(x)\right| .
\end{aligned}
$$

Hence, taking the infimum on the right hand side over all $g \in W^{2}$ and using (3.8), we get the required result. 
Theorem 5 Let $f \in C_{2}[0, \infty), q_{n} \in(0,1)$ and $\omega_{a+1}(f, \delta)$ be its modulus of continuity on the finite interval $[0, a+1] \subset[0, \infty)$, where $a>0$. Then, for every $n>2$,

$$
\left|B_{n, q_{n}}^{(\alpha, \beta)}(f ; x)-f(x)\right| \leq 4 M_{f}\left(1+a^{2}\right) \gamma_{n, q_{n}}(x)+2 \omega_{a+1}\left(f, \sqrt{\gamma_{n, q_{n}}(x)}\right),
$$

where $\gamma_{n, q_{n}}(x)$ is defined in Remark 1.

Proof From [24], for $x \in[0, a]$ and $t \in[0, \infty)$, we get

$$
|f(t)-f(x)| \leq 4 M_{f}\left(1+a^{2}\right)(t-x)^{2}+\left(1+\frac{|t-x|}{\delta}\right) \omega_{a+1}(f, \delta), \quad \delta>0 .
$$

Thus, by applying the Cauchy-Schwarz inequality, we have

$$
\begin{aligned}
\left|B_{n, q_{n}}^{(\alpha, \beta)}(f ; x)-f(x)\right| \leq & 4 M_{f}\left(1+a^{2}\right)\left(B_{n, q_{n}}^{(\alpha, \beta)}(t-x)^{2} ; x\right) \\
& +\omega_{a+1}(f, \delta)\left(1+\frac{1}{\delta}\left(B_{n, q_{n}}^{(\alpha, \beta)}(t-x)^{2} ; x\right)^{\frac{1}{2}}\right) \\
= & 4 M_{f}\left(1+a^{2}\right) \gamma_{n, q_{n}}(x)+2 \omega_{a+1}\left(f, \sqrt{\gamma_{n, q_{n}}(x)}\right),
\end{aligned}
$$

on choosing $\delta=\sqrt{\gamma_{n, q_{n}}(x)}$. This completes the proof of the theorem.

\subsection{Weighted approximation}

Let $C_{2}^{*}[0, \infty):=\left\{f \in C_{2}[0, \infty): \lim _{x \rightarrow \infty} \frac{|f(x)|}{1+x^{2}}<\infty\right\}$. Throughout the section, we assume that $\left\{q_{n}\right\}$ is a sequence in $(0,1)$ such that $q_{n} \rightarrow 1$.

Theorem 6 For each $f \in C_{2}^{*}[0, \infty)$, we have

$$
\lim _{n \rightarrow \infty}\left\|B_{n, q_{n}}^{(\alpha, \beta)}(f)-f\right\|_{2}=0
$$

Proof Making use of the Korovkin type theorem on weighted approximation [25], we see that it is sufficient to verify the following three conditions:

$$
\lim _{n \rightarrow \infty}\left\|B_{n, q_{n}}^{(\alpha, \beta)}\left(t^{k} ; x\right)-x^{k}\right\|_{2}=0, \quad k=0,1,2
$$

Since $B_{n, q_{n}}^{(\alpha, \beta)}(1 ; x)=1$, the condition in (3.14) holds for $k=0$.

By Lemma 2, we have for $n>1$

$$
\begin{aligned}
& \left\|B_{n, q_{n}}^{(\alpha, \beta)}(t ; x)-x\right\|_{2} \\
& \quad \leq\left|\frac{[n]_{q_{n}} q_{n}^{n-1}-\beta[n-1]_{q_{n}}}{\left([n]_{q_{n}}+\beta\right)[n-1]_{q_{n}}}\right| \sup _{x \in[0, \infty)} \frac{x}{1+x^{2}}+\frac{\alpha}{[n]_{q_{n}}+\beta} \sup _{x \in[0, \infty)} \frac{1}{1+x^{2}} \\
& \quad \leq\left|\frac{[n]_{q_{n}} q_{n}^{n-1}-\beta[n-1]_{q_{n}}}{\left([n]_{q_{n}}+\beta\right)[n-1]_{q_{n}}}\right|+\frac{\alpha}{[n]_{q_{n}}+\beta},
\end{aligned}
$$

which implies that the condition in (3.14) holds for $k=1$. 
Similarly, we can write for $n>2$

$$
\begin{aligned}
\left\|B_{n, q_{n}}^{(\alpha, \beta)}\left(t^{2} ; x\right)-x^{2}\right\|_{2} \leq & \left|\frac{q_{n}[n]_{q_{n}}^{2}}{[n-1]_{q_{n}}[n-2]_{q_{n}}}+\frac{2 \alpha}{[n-1]_{q_{n}}}-1\right| \\
& +\frac{[2]_{q_{n}}[n]_{q_{n}}}{q_{n}[n-1]_{q_{n}}[n-2]_{q_{n}}}+\frac{\alpha^{2}}{\left([n]_{q_{n}}+\beta\right)^{2}}
\end{aligned}
$$

which implies that $\lim _{n \rightarrow \infty}\left\|B_{n, q_{n}}^{(\alpha, \beta)}\left(t^{2} ; x\right)-x^{2}\right\|_{2}=0$, (3.14) holds for $k=2$.

Now, we present a weighted approximation theorem for functions in $C_{2}^{*}[0, \infty)$. Such type of results are proved in [26] for classical Szász operators.

Theorem 7 For each $f \in C_{2}^{*}[0, \infty)$ and $\alpha>0$, we have

$$
\lim _{n \rightarrow \infty} \sup _{x \in[0, \infty)} \frac{\left|B_{n, q_{n}}^{(\alpha, \beta)}(f ; x)-f(x)\right|}{\left(1+x^{2}\right)^{1+\alpha}}=0 .
$$

Proof Let $x_{0} \in[0, \infty)$ be arbitrary but fixed. Then

$$
\begin{aligned}
\sup _{x \in[0, \infty)} \frac{\left|B_{n, q_{n}}^{(\alpha, \beta)}(f ; x)-f(x)\right|}{\left(1+x^{2}\right)^{1+\alpha}} \leq & \sup _{x \leq x_{0}} \frac{\left|B_{n, q_{n}}^{(\alpha, \beta)}(f ; x)-f(x)\right|}{\left(1+x^{2}\right)^{1+\alpha}}+\sup _{x>x_{0}} \frac{\left|B_{n, q_{n}}^{(\alpha, \beta)}(f ; x)-f(x)\right|}{\left(1+x^{2}\right)^{1+\alpha}} \\
\leq & \left\|B_{n, q_{n}}^{(\alpha, \beta)}(f)-f\right\|_{C\left[0, x_{0}\right]}+\|f\|_{2} \sup _{x>x_{0}} \frac{\left|B_{n, q_{n}}^{(\alpha, \beta)}\left(1+t^{2} ; x\right)\right|}{\left(1+x^{2}\right)^{1+\alpha}} \\
& +\sup _{x>x_{0}} \frac{|f(x)|}{\left(1+x^{2}\right)^{1+\alpha}} .
\end{aligned}
$$

Since $|f(x)| \leq\|f\|_{2}\left(1+x^{2}\right)$, we have $\sup _{x>x_{0}} \frac{|f(x)|}{\left(1+x^{2}\right)^{1+\alpha}} \leq \frac{\|f\|_{2}}{\left(1+x_{0}^{2}\right)^{\alpha}}$.

Let $\epsilon>0$ be arbitrary. We can choose $x_{0}$ to be so large that

$$
\frac{\|f\|_{2}}{\left(1+x_{0}^{2}\right)^{\alpha}}<\frac{\epsilon}{3}
$$

In view of Theorem 1 , we obtain

$$
\|f\|_{2} \lim _{n \rightarrow \infty} \frac{\left|B_{n, q_{n}}^{(\alpha, \beta)}\left(1+t^{2} ; x\right)\right|}{\left(1+x^{2}\right)^{1+\alpha}}=\frac{1+x^{2}}{\left(1+x^{2}\right)^{1+\alpha}}\|f\|_{2}=\frac{\|f\|_{2}}{\left(1+x^{2}\right)^{\alpha}} \leq \frac{\|f\|_{2}}{\left(1+x_{0}^{2}\right)^{\alpha}}<\frac{\epsilon}{3}
$$

Using Theorem 5, we can see that the first term of the inequality (3.15), implies that

$$
\left\|B_{n, q_{n}}^{(\alpha, \beta)}(f)-f\right\|_{C\left[0, x_{0}\right]}<\frac{\epsilon}{3}, \quad \text { as } n \rightarrow \infty
$$

Combining (3.16)-(3.18), we get the desired result.

For $f \in C_{2}^{*}[0, \infty)$, the weighted modulus of continuity is defined as

$$
\Omega_{2}(f, \delta)=\sup _{x \geq 0,0<h \leq \delta} \frac{|f(x+h)-f(x)|}{1+(x+h)^{2}} .
$$


Lemma 4 [27] If $\in C_{2}^{*}[0, \infty)$ then

(i) $\Omega_{2}(f, \delta)$ is monotone increasing function of $\delta$,

(ii) $\lim _{\delta \rightarrow 0^{+}} \Omega_{2}(f, \delta)=0$,

(iii) for any $\lambda \in[0, \infty), \Omega_{2}(f, \lambda \delta) \leq(1+\lambda) \Omega_{2}(f, \delta)$.

Theorem 8 If $f \in C_{2}^{*}[0, \infty)$, then for sufficiently large $n$ we have

$$
\left|B_{n, q_{n}}^{(\alpha, \beta)}(f ; x)-f(x)\right| \leq K\left(1+x^{2+\lambda}\right) \Omega_{2}\left(f, \delta_{n}\right), \quad x \in[0, \infty),
$$

where $\lambda \geq 1, \delta_{n}=\max \left\{\alpha_{n}, \beta_{n}, \gamma_{n}\right\}, \alpha_{n}, \beta_{n}, \gamma_{n}$ being

$$
\begin{aligned}
& \left(\frac{q_{n}[n]_{q_{n}}^{4}+2 \alpha[n-2]_{q_{n}}[n]_{q_{n}}^{2}}{[n-1]_{q_{n}}[n-2]_{q_{n}}\left([n]_{q_{n}}+\beta\right)^{2}}+1\right), \quad\left(\frac{2[n]_{q_{n}}^{3}}{q_{n}[n-1]_{q_{n}}[n-2]_{q_{n}}\left([n]_{q_{n}}+\beta\right)^{2}}\right) \text { and } \\
& \frac{\alpha^{2}}{\left([n]_{q_{n}}+\beta\right)^{2}}
\end{aligned}
$$

respectively, and $K$ is a positive constant independent of $f$ and $n$.

Proof From the definition of $\Omega_{2}(f, \delta)$ and Lemma 4, we have

$$
\begin{aligned}
|f(t)-f(x)| & \leq\left(1+(x+|t-x|)^{2}\right)\left(1+\frac{|t-x|}{\delta}\right) \Omega_{2}(f, \delta) \\
& \leq\left(1+(2 x+t)^{2}\right)\left(1+\frac{|t-x|}{\delta}\right) \Omega_{2}(f, \delta) \\
& :=\phi_{x}(t)\left(1+\frac{1}{\delta} \psi_{x}(t)\right) \Omega_{2}(f, \delta),
\end{aligned}
$$

where $\phi_{x}(t)=1+(2 x+t)^{2}$ and $\psi_{x}(t)=|t-x|$. Then we obtain

$$
\left|B_{n, q_{n}}^{(\alpha, \beta)}(f ; x)-f(x)\right| \leq\left(B_{n, q_{n}}^{(\alpha, \beta)}\left(\phi_{x} ; x\right)+\frac{1}{\delta_{n}} B_{n, q_{n}}^{(\alpha, \beta)}\left(\phi_{x} \psi_{x} ; x\right)\right) \Omega_{2}\left(f, \delta_{n}\right) .
$$

Now, applying the Cauchy-Schwarz inequality to the second term on the right hand side, we get

$$
\begin{aligned}
& \left|B_{n, q_{n}}^{(\alpha, \beta)}(f ; x)-f(x)\right| \\
& \quad \leq\left(B_{n, q_{n}}^{(\alpha, \beta)}\left(\phi_{x} ; x\right)+\frac{1}{\delta_{n}} \sqrt{B_{n, q_{n}}^{(\alpha, \beta)}\left(\phi_{x}^{2} ; x\right)} \sqrt{B_{n, q_{n}}^{(\alpha, \beta)}\left(\psi_{x}^{2} ; x\right)}\right) \Omega_{2}\left(f, \delta_{n}\right) .
\end{aligned}
$$

From Lemma 2,

$$
\begin{aligned}
\frac{1}{1+x^{2}} B_{n, q_{n}}^{(\alpha, \beta)}\left(1+t^{2} ; x\right)= & \frac{1}{1+x^{2}}+\left(\frac{q_{n}[n]_{q_{n}}^{4}+2 \alpha[n-2]_{q_{n}}[n]_{q_{n}}^{2}}{[n-1]_{q_{n}}[n-2]_{q_{n}}\left([n]_{q_{n}}+\beta\right)^{2}}\right) \frac{x^{2}}{1+x^{2}} \\
& +\frac{[2]_{q_{n}}[n]_{q_{n}}^{3}}{q_{n}[n-1]_{q_{n}}[n-2]_{q_{n}}\left([n]_{q_{n}}+\beta\right)^{2}} \frac{x}{1+x^{2}}+\frac{\alpha^{2}}{\left([n]_{q_{n}}+\beta\right)^{2}} \frac{1}{1+x^{2}} \\
\leq & 1+C_{1}, \quad \text { for sufficiently large } n
\end{aligned}
$$

where $C_{1}$ is a positive constant. 
From (3.20), there exists a positive constant $K_{1}$ such that $B_{n, q_{n}}^{(\alpha, \beta)}\left(\phi_{x} ; x\right) \leq K_{1}\left(1+x^{2}\right)$, for sufficiently large $n$.

Proceeding similarly, $\frac{1}{1+x^{4}} B_{n, q_{n}}^{(\alpha, \beta)}\left(1+t^{4} ; x\right) \leq 1+C_{2}$, for sufficiently large $n$, where $C_{2}$ is a positive constant.

So there exists a positive constant $K_{2}$, such that $\sqrt{B_{n, q_{n}}^{(\alpha, \beta)}\left(\phi_{x}^{2} ; x\right)} \leq K_{2}\left(1+x^{2}\right)$, where $x \in$ $[0, \infty)$ and $n$ is large enough. Also, we get

$$
\begin{aligned}
B_{n, q_{n}}^{(\alpha, \beta)}\left(\psi_{x}^{2} ; x\right)= & \left\{\frac{q_{n}[n]_{q_{n}}^{4}+2 \alpha[n-2]_{q_{n}}[n]_{q_{n}}^{2}}{[n-1]_{q_{n}}[n-2]_{q_{n}}\left([n]_{q_{n}}+\beta\right)^{2}}+1-\frac{2[n]_{q_{n}}^{2}}{\left([n]_{q_{n}}+\beta\right)[n-1]_{q_{n}}}\right\} x^{2} \\
& +\left\{\frac{2[n]_{q_{n}}^{3}}{q_{n}[n-1]_{q_{n}}[n-2]_{q_{n}}\left([n]_{q_{n}}+\beta\right)^{2}}-\frac{2 \alpha}{[n]_{q_{n}}+\beta}\right\} x+\frac{\alpha^{2}}{\left([n]_{q_{n}}+\beta\right)^{2}} \\
\leq & \alpha_{n} x^{2}+\beta_{n} x+\gamma_{n} .
\end{aligned}
$$

Hence, from (3.19), we have

$$
\left|B_{n, q_{n}}^{(\alpha, \beta)}(f ; x)-f(x)\right| \leq\left(1+x^{2}\right)\left(K_{1}+\frac{1}{\delta_{n}} K_{2} \sqrt{\alpha_{n} x^{2}+\beta_{n} x+\gamma_{n}}\right) \Omega_{2}\left(f, \delta_{n}\right) .
$$

If we take $\delta_{n}=\max \left\{\alpha_{n}, \beta_{n}, \gamma_{n}\right\}$, then we get

$$
\begin{aligned}
\left|B_{n, q_{n}}^{(\alpha, \beta)}(f ; x)-f(x)\right| & \leq\left(1+x^{2}\right)\left(K_{1}+K_{2} \sqrt{x^{2}+x+1}\right) \Omega_{2}\left(f, \delta_{n}\right) \\
& \leq K_{3}\left(1+x^{2+\lambda}\right) \Omega_{2}\left(f, \delta_{n}\right), \quad \text { for sufficiently large } n \text { and } x \in[0, \infty)
\end{aligned}
$$

Hence, the proof is completed.

\subsection{Point-wise estimates}

In this section, we establish some point-wise estimates of the rate of convergence of the operators $B_{n, q}^{(\alpha, \beta)}$. First, we give the relationship between the local smoothness of $f$ and local approximation.

We know that a function $f \in C[0, \infty)$ is in $\operatorname{Lip}_{M} \eta$ on $E, \eta \in(0,1], E$ be any bounded subset of the interval $[0, \infty)$ if it satisfies the condition

$$
|f(t)-f(x)| \leq M|t-x|^{\eta}, \quad t \in[0, \infty) \text { and } x \in E,
$$

where $M$ is a constant depending only on $\alpha$ and $f$.

Theorem 9 Let $f \in C[0, \infty) \cap \operatorname{Lip}_{M} \eta, E \subset[0, \infty)$ and $\alpha \in(0,1]$. Then we have

$$
\left|B_{n, q}^{(\alpha, \beta)}(f ; x)-f(x)\right| \leq M\left(\gamma_{n, q}^{\eta / 2}(x)+2 d^{\eta}(x, E)\right), \quad x \in[0, \infty),
$$

where $M$ is a constant depending on $\eta$ and $f$, and $d(x, E)$ is the distance between $x$ and $E$ defined as

$$
d(x, E)=\inf \{|t-x|: t \in E\} .
$$


Proof Let $\bar{E}$ be the closure of $E$ in $[0, \infty)$. Then there exists at least one point $x_{0} \in \bar{E}$ such that

$$
d(x, E)=\left|x-x_{0}\right|
$$

By our hypothesis and the monotonicity of $B_{n, q}^{(\alpha, \beta)}$, we get

$$
\begin{aligned}
\left|B_{n, q}^{(\alpha, \beta)}(f ; x)-f(x)\right| & \leq B_{n, q}^{(\alpha, \beta)}\left(\left|f(t)-f\left(x_{0}\right)\right| ; x\right)+B_{n, q}^{(\alpha, \beta)}\left(\left|f(x)-f\left(x_{0}\right)\right| ; x\right) \\
& \leq M\left\{B_{n, q}^{(\alpha, \beta)}\left(\left|t-x_{0}\right|^{\eta} ; x\right)+\left|x-x_{0}\right|^{\eta}\right\} \\
& \leq M\left\{B_{n, q}^{(\alpha, \beta)}\left(|t-x|^{\eta} ; x\right)+2\left|x-x_{0}\right|^{\eta}\right\} .
\end{aligned}
$$

Now, applying Hölder's inequality with $p=\frac{2}{\eta}$ and $\frac{1}{q}=1-\frac{1}{p}$, we obtain

$$
\left|B_{n, q}^{(\alpha, \beta)}(f ; x)-f(x)\right| \leq M\left\{\left[B_{n, q}^{(\alpha, \beta)}\left(|t-x|^{2} ; x\right)\right]^{\eta / 2}+2 d^{\eta}(x, E)\right\}
$$

from which the desired result immediate.

Next, we obtain the local direct estimate of the operators defined in (1.3), using the Lipschitz-type maximal function of order $\eta$ introduced by Lenze [28] as

$$
\widetilde{\omega}_{\eta}(f, x)=\sup _{t \neq x, t \in[0, \infty)} \frac{|f(t)-f(x)|}{|t-x|^{\eta}}, \quad x \in[0, \infty) \text { and } \eta \in(0,1] .
$$

Theorem 10 Let $f \in C_{B}[0, \infty)$ and $0<\eta \leq 1$. Then, for all $x \in[0, \infty)$ we have

$$
\left|B_{n, q}^{(\alpha, \beta)}(f ; x)-f(x)\right| \leq \widetilde{\omega}_{\eta}(f, x) \gamma_{n, q}^{\eta / 2}(x) .
$$

Proof From (3.21), we have

$$
\left|B_{n, q}^{(\alpha, \beta)}(f ; x)-f(x)\right| \leq \widetilde{\omega}_{\eta}(f, x) B_{n, q}^{(\alpha, \beta)}\left(|t-x|^{\eta} ; x\right) .
$$

Applying Hölder's inequality with $p=\frac{2}{\eta}$ and $\frac{1}{q}=1-\frac{1}{p}$, we get

$$
\left|B_{n, q}^{(\alpha, \beta)}(f ; x)-f(x)\right| \leq \widetilde{\omega}_{\eta}(f, x) B_{n, q}^{(\alpha, \beta)}\left((t-x)^{2} ; x\right)^{\frac{\eta}{2}} \leq \widetilde{\omega}_{\eta}(f, x) \gamma_{n, q}^{\eta / 2}(x) .
$$

Thus, the proof is completed.

\section{Statistical convergence}

Let $A=\left(a_{n k}\right)$ be a non-negative infinite summability matrix. For a given sequence $x:=(x)_{n}$, the $A$-transform of $\mathrm{x}$ denoted by $A x:(A x)_{n}$ is defined as

$$
(A x)_{n}=\sum_{k=1}^{\infty} a_{n k} x_{k}
$$

provided the series converges for each $n$. A is said to be regular if $\lim _{n}(A x)_{n}=L$ whenever $\lim _{n}(x)_{n}=L$. Then $x=(x)_{n}$ is said to be $A$-statistically convergent to $L$ i.e. $s t_{A}-\lim _{n}(x)_{n}=L$ 
if for every $\epsilon>0, \lim _{n} \sum_{k:\left|x_{k}-L\right| \geq \epsilon} a_{n k}=0$. If we replace $A$ by $C_{1}$ then $A$ is a Cesaro matrix of order one and $A$-statistical convergence is reduced to the statistical convergence. Similarly, if $A=I$, the identity matrix, then $A$-statistical convergence is called ordinary convergence. Kolk [29] proved that statistical convergence is better than ordinary convergence. In this direction, significant contributions have been made by (cf. [19, 20, 30-37] etc.).

Let $q_{n} \in(0,1)$ be a sequence such that

$$
s t_{A}-\lim _{n} q_{n}=1, \quad s t_{A}-\lim _{n} q_{n}^{n}=a \quad(a<1) \quad \text { and } \quad s t_{A}-\lim _{n} \frac{1}{[n]_{q_{n}}}=0 .
$$

Theorem 11 Let $A=\left(a_{n k}\right)$ be a non-negative regular summability matrix and $\left(q_{n}\right)$ be a sequence satisfying (4.1). Then, for any compact set $K \subset[0, \infty)$ and for each function $f \in$ $C(K)$, we have

$$
s t_{A}-\lim _{n}\left\|B_{n, q_{n}}^{(\alpha, \beta)}(f ; \cdot)-f\right\|=0 .
$$

Proof Let $x_{0}=\max _{x \in K} x$. From Lemma $2, s t_{A}-\lim _{n}\left\|B_{n, q_{n}}^{(\alpha, \beta)}\left(e_{0} ; \cdot\right)-e_{0}\right\|=0$. Again, by Lemma 2, we have

$$
\sup _{x \in K}\left|B_{n, q_{n}}^{(\alpha, \beta)}\left(e_{1} ; x\right)-e_{1}(x)\right| \leq\left|\frac{[n]_{q_{n}}^{2}}{\left([n]_{q_{n}}+\beta\right)[n-1]_{q_{n}}}-1\right| x_{0}+\frac{\alpha}{[n]_{q_{n}}+\beta} .
$$

For $\epsilon>0$, let us define the following sets:

$$
\begin{aligned}
& G:=\left\{k:\left\|B_{k, q_{k}}^{(\alpha, \beta)}\left(e_{1} ; \cdot\right)-e_{1}\right\| \geq \epsilon\right\}, \\
& G_{1}:=\left\{k:\left|\frac{[k]_{q_{k}}^{2}}{\left([k]_{q_{k}}+\beta\right)[k-1]_{q_{k}}}-1\right| \geq \frac{\epsilon}{2}\right\}, \\
& G_{2}:=\left\{k: \frac{\alpha}{[k]_{q_{k}}+\beta} \geq \frac{\epsilon}{2}\right\},
\end{aligned}
$$

which implies that $G \subseteq G_{1} \cup G_{2}$ and hence for all $n \in \mathbb{N}$, we obtain

$$
\sum_{k \in G} a_{n k} \leq \sum_{k \in G_{1}} a_{n k}+\sum_{k \in G_{2}} a_{n k}
$$

Hence, taking the limit as $n \rightarrow \infty$, we have $s t_{A}-\lim _{n}\left\|B_{n, q_{n}}^{(\alpha, \beta)}\left(e_{1} ; \cdot\right)-e_{1}\right\|=0$.

Similarly, by using Lemma 2 , we have

$$
\begin{aligned}
\sup _{x \in K}\left|B_{n, q_{n}}^{(\alpha, \beta)}\left(e_{2} ; x\right)-e_{2}(x)\right| \leq & \left|\frac{q_{n}[n]_{q_{n}}^{4}+2 \alpha[n-2]_{q_{n}}}{[n-1]_{q_{n}}[n-2]_{q_{n}}\left([n]_{q_{n}}+\beta\right)^{2}}-1\right| x_{0}^{2} \\
& +\frac{[2]_{q_{n}}[n]_{q_{n}}^{3}}{q_{n}[n-1]_{q_{n}}[n-1]_{q_{n}}\left([n]_{q_{n}}+\beta\right)^{2}} x_{0}+\frac{\alpha^{2}}{\left([n]_{q_{n}}+\beta\right)^{2}}
\end{aligned}
$$

Now, let us define the following sets:

$$
M:=\left\{k:\left\|B_{k, q_{k}}^{(\alpha, \beta)}\left(e_{2} ; \cdot\right)-e_{2}\right\| \geq \epsilon\right\},
$$




$$
\begin{aligned}
& M_{1}:=\left\{k:\left|\frac{q_{k}[k]_{q_{k}}^{4}+2 \alpha[k-2]_{q_{k}}}{[k-1]_{q_{k}}[k-2]_{q_{k}}\left([k]_{q_{k}}+\beta\right)^{2}}-1\right| \geq \frac{\epsilon}{3}\right\}, \\
& M_{2}:=\left\{k: \frac{[2]_{q_{k}}[k]_{q_{k}}^{3}}{q_{k}[k-1]_{q_{k}}[k-1]_{q_{k}}\left[[k]_{q_{k}}+\beta\right)^{2}} \geq \frac{\epsilon}{3}\right\}, \\
& M_{3}:=\left\{k: \frac{\alpha^{2}}{\left([k]_{q_{k}}+\beta\right)^{2}} \geq \frac{\epsilon}{3}\right\} .
\end{aligned}
$$

Then we obtain $M \subseteq M_{1} \cup M_{2} \cup M_{3}$, which implies that

$$
\sum_{k \in M} a_{n k} \leq \sum_{k \in M_{1}} a_{n k}+\sum_{k \in M_{2}} a_{n k}+\sum_{k \in M_{3}} a_{n k}
$$

Thus, as $n \rightarrow \infty$ we get $s t_{A}-\lim _{n}\left\|B_{n, q_{n}}^{(\alpha, \beta)}\left(e_{2} ; \cdot\right)-e_{2}\right\|=0$. This completes the proof.

Theorem 12 Let $A=\left(a_{n k}\right)$ be a non-negative regular summability matrix and $\left(q_{n}\right)$ be a sequence in $(0,1)$ satisfying (4.1). Let the operators $B_{n, q_{n}}^{(\alpha, \beta)}, n \in \mathbb{N}$, be defined as in (1.3). Then, for each function $f \in C_{2}[0, \infty)$, we have

$$
s t_{A}-\lim _{n}\left\|B_{n, q_{n}}^{(\alpha, \beta)}(f ; \cdot)-f\right\|_{\rho}=0,
$$

where $\rho(x)=1+x^{2+\lambda}, \lambda>0$.

Proof From [31, p.191, Theorem 3], it is sufficient to prove that $s t_{A}-\lim _{n}\left\|B_{n, q_{n}}^{(\alpha, \beta)}\left(e_{i} ; \cdot\right)-e_{i}\right\|_{2}=$ 0 , where $e_{i}(x)=x^{i}, i=0,1,2$.

From Lemma $2, s t_{A}-\lim _{n}\left\|B_{n, q_{n}}^{(\alpha, \beta)}\left(e_{0} ; \cdot\right)-e_{0}\right\|_{2}=0$ holds.

Again using Lemma 2, we have

$$
\begin{aligned}
\left\|B_{n, q_{n}}^{(\alpha, \beta)}\left(e_{1} ; \cdot\right)-e_{1}\right\|_{2} & \leq \sup _{x \in[0, \infty)}\left\{\frac{x}{\left(1+x^{2}\right)}\left|\frac{[n]_{q_{n}}^{2}}{\left([n]_{q_{n}}+\beta\right)[n-1]_{q_{n}}}-1\right|+\frac{1}{\left(1+x^{2}\right)} \frac{\alpha}{\left([n]_{q_{n}}+\beta\right)}\right\} \\
& \leq\left|\frac{[n]_{q_{n}}^{2}}{\left([n]_{q_{n}}+\beta\right)[n-1]_{q_{n}}}-1\right|+\frac{\alpha}{\left([n]_{q_{n}}+\beta\right)}
\end{aligned}
$$

For each $\epsilon>0$, let us define the following sets:

$$
\begin{aligned}
& G:=\left\{k:\left\|B_{k, q_{k}}^{(\alpha, \beta)}\left(e_{1} ; \cdot\right)-e_{1}\right\| \geq \epsilon\right\}, \\
& G_{1}:=\left\{k:\left|\frac{[k]_{q_{k}}^{2}}{\left([k]_{q_{k}}+\beta\right)[k-1]_{q_{k}}}-1\right| \geq \frac{\epsilon}{2}\right\}, \\
& G_{2}:=\left\{k: \frac{\alpha}{[k]_{q_{k}}+\beta} \geq \frac{\epsilon}{2}\right\},
\end{aligned}
$$

which yields $G \subseteq G_{1} \cup G_{2}$ in view of (4.2) and therefore for all $n \in \mathbb{N}$, we have

$$
\sum_{k \in G} a_{n k} \leq \sum_{k \in G_{1}} a_{n k}+\sum_{k \in G_{2}} a_{n k}
$$


Hence, on taking the limit as $n \rightarrow \infty, s t_{A}-\lim _{n}\left\|B_{n, q_{n}}^{(\alpha, \beta)}\left(e_{1} ; \cdot\right)-e_{1}\right\|_{2}=0$. Proceeding similarly,

$$
\begin{aligned}
\left\|B_{n, q_{n}}^{(\alpha, \beta)}\left(e_{2} ; \cdot\right)-e_{2}\right\|_{2} \leq & \left|\frac{q_{n}[n]_{q_{n}}^{4}+2 \alpha[n-2]_{q_{n}}}{[n-1]_{q_{n}}[n-2]_{q_{n}}\left([n]_{q_{n}}+\beta\right)^{2}}-1\right| \\
& +\frac{[2]_{q_{n}}[n]_{q_{n}}^{3}}{q_{n}[n-1]_{q_{n}}[n-1]_{q_{n}}\left([n]_{q_{n}}+\beta\right)^{2}}+\frac{\alpha^{2}}{\left([n]_{q_{n}}+\beta\right)^{2}}
\end{aligned}
$$

Now, let us define the following sets:

$$
\begin{aligned}
& M:=\left\{k:\left\|B_{k, q_{k}}^{(\alpha, \beta)}\left(e_{2} ; \cdot\right)-e_{2}\right\| \geq \epsilon\right\}, \\
& M_{1}:=\left\{k:\left|\frac{q_{k}[k]_{q_{k}}^{4}+2 \alpha[k-2]_{q_{k}}}{[k-1]_{q_{k}}[k-2]_{q_{k}}\left([k]_{q_{k}}+\beta\right)^{2}}-1\right| \geq \frac{\epsilon}{3}\right\}, \\
& M_{2}:=\left\{k: \frac{[2]_{q_{k}}[k]_{q_{k}}^{3}}{q_{k}[k-1]_{q_{k}}[k-1]_{q_{k}}\left([k]_{q_{k}}+\beta\right)^{2}} \geq \frac{\epsilon}{3}\right\}, \\
& M_{3}:=\left\{k: \frac{\alpha^{2}}{\left([k]_{q_{k}}+\beta\right)^{2}} \geq \frac{\epsilon}{3}\right\} .
\end{aligned}
$$

Then we obtain $M \subseteq M_{1} \cup M_{2} \cup M_{3}$, which implies that

$$
\sum_{k \in M} a_{n k} \leq \sum_{k \in M_{1}} a_{n k}+\sum_{k \in M_{2}} a_{n k}+\sum_{k \in M_{3}} a_{n k} .
$$

Hence, taking the limit as $n \rightarrow \infty$ we get $s t_{A}-\lim _{n}\left\|B_{n, q_{n}}^{(\alpha, \beta)}\left(e_{2} ; \cdot\right)-e_{2}\right\|_{2}=0$. This completes the proof of the theorem.

\section{Better estimates}

It is well known that the classical Bernstein polynomials preserve constant as well as linear functions. To make the convergence faster, King [38] proposed an approach to modify the Bernstein polynomials, so that the sequence preserves test functions $e_{0}$ and $e_{2}$, where $e_{i}(t)=t^{i}, i=0,1,2$. As the operator $B_{n, q}^{(\alpha, \beta)}(f ; x)$ defined in (1.3) reproduces only constant functions, this motivated us to propose the modification of this operator, so that it can preserve constant as well as linear functions.

The modification of the operators given in (1.3) is defined as

$$
\begin{aligned}
\tilde{B}_{n, q}^{(\alpha, \beta)}(f ; x)= & \sum_{\nu=1}^{\infty} q_{n, \nu}\left(r_{n}^{q}(x)\right) q^{\nu-1} \int_{0}^{\infty / A} b_{n, \nu-1}(q, t) f\left(\frac{q^{\nu}[n]_{q} t+\alpha}{[n]_{q}+\beta}\right) d_{q} t \\
& +e_{q}\left(-[n]_{q} r_{n}^{q}(x)\right) f\left(\frac{\alpha}{[n]_{q}+\beta}\right)
\end{aligned}
$$

where $r_{n}^{q}(x)=\frac{\left([n]_{q}+\beta\right)[n-1]_{q} x-\alpha[n-1]_{q}}{[n]_{q}^{2}}$ for $x \in I_{n}=\left[\frac{\alpha}{[n]_{q}+\beta}, \infty\right)$ and $n>1$.

Lemma 5 For each $x \in I_{n}$, by simple computations, we have

(1) $\tilde{B}_{n, q}^{(\alpha, \beta)}(1 ; x)=1$;

(2) $\tilde{B}_{n, q}^{(\alpha, \beta)}(t ; x)=x$; 
(3) $\tilde{B}_{n, q}^{(\alpha, \beta)}\left(t^{2} ; x\right)=\frac{[n-1]_{q}\left(q[n]_{q}^{2}+2 \alpha[n-2]_{q}\right)}{[n-2]_{q}[n]_{q}^{2}} x^{2}+\frac{[2]_{q}[n]_{q}^{3}-2 q \alpha[n-1]_{q}\left(q[n]_{q}^{2}+2 \alpha[n-2]_{q}\right)}{q\left([n]_{q}+\beta\right)[n-2]_{q}[n]_{q}^{2}} x+$ $\frac{\alpha^{2}[n]_{q}^{2}\left(q[n-1]_{q}+[n-2]_{q}\right)+2 \alpha^{3}[n-1]_{q}[n-2]_{q}}{\left([n]_{q}+\beta\right)^{2}[n]_{q}^{2}[n-2]_{q}}$.

Consequently, for each $x \in I_{n}$, we have the following equalities:

$$
\begin{aligned}
\tilde{B}_{n, q}^{(\alpha, \beta)}(t-x ; x)=0, & \\
\tilde{B}_{n, q}^{(\alpha, \beta)}\left((t-x)^{2} ; x\right)= & \frac{[n]_{q}^{2}\left(q[n-1]_{q}-[n-2]_{q}\right)+2 \alpha[n-1]_{q}[n-2]_{q}}{[n-2]_{q}[n]_{q}^{2}} x^{2} \\
& +\frac{[2]_{q}[n]_{q}^{3}-2 q \alpha[n-1]_{q}\left(q[n]_{q}^{2}-2 \alpha[n-2]_{q}\right)}{q\left([n]_{q}+\beta\right)[n-2]_{q}[n]_{q}^{2}} x \\
& +\frac{\alpha^{2}[n]_{q}^{2}\left(q[n-1]_{q}+[n-2]_{q}\right)+2 \alpha[n-1]_{q}[n-2]_{q}}{\left([n]_{q}+\beta\right)^{2}[n]_{q}^{2}[n-2]_{q}} \\
=: & \xi_{n}(x),
\end{aligned}
$$

say.

Theorem 13 Let $f \in C_{B}[0, \infty)$ and $x \in I_{n}$. Then there exists a positive constant $C$ such that

$$
\left|\tilde{B}_{n, q}^{(\alpha, \beta)}(f ; x)-f(x)\right| \leq C \omega_{2}\left(f, \sqrt{\xi_{n}(x)}\right)
$$

where $\xi_{n}(x)$ is given by (5.1).

Proof Let $g \in W^{2}, x \in I_{n}$ and $t \in[0, \infty)$. Using Taylor's expansion we have

$$
g(t)=g(x)+(t-x) g^{\prime}(x)+\int_{x}^{t}(t-u) g^{\prime \prime}(u) d u
$$

Applying $\tilde{B}_{n, q}^{(\alpha, \beta)}$ on both sides and using Lemma 5, we get

$$
\tilde{B}_{n, q}^{(\alpha, \beta)}(g ; x)-g(x)=\tilde{B}_{n, q}^{(\alpha, \beta)}((t-x) ; x) g^{\prime}(x)+\tilde{B}_{n, q}^{(\alpha, \beta)}\left(\int_{x}^{t}(t-u) g^{\prime \prime}(u) d u ; x\right) .
$$

Obviously, we have $\left|\int_{x}^{t}(t-u) g^{\prime \prime}(u) d u\right| \leq(t-x)^{2}\left\|g^{\prime \prime}\right\|$. Therefore

$$
\left|\tilde{B}_{n, q}^{(\alpha, \beta)}(g ; x)-g(x)\right| \leq \tilde{B}_{n, q}^{(\alpha, \beta)}\left((t-x)^{2} ; x\right)\left\|g^{\prime \prime}\right\|=\xi_{n}(x)\left\|g^{\prime \prime}\right\|
$$

Since $\left|\tilde{B}_{n, q}^{(\alpha, \beta)}(f ; x)\right| \leq\|f\|$, we get

$$
\begin{aligned}
\left|\tilde{B}_{n, q}^{(\alpha, \beta)}(f ; x)-f(x)\right| & \leq\left|\tilde{B}_{n, q}^{(\alpha, \beta)}(f-g ; x)\right|+|(f-g)(x)|+\left|\tilde{B}_{n, q}^{(\alpha, \beta)}(g ; x)-g(x)\right| \\
& \leq 2\|f-g\|+\xi_{n}(x)\left\|g^{\prime \prime}\right\| .
\end{aligned}
$$

Finally, taking the infimum over all $g \in W^{2}$ and using (3.7)-(3.9) we obtain

$$
\left|\tilde{B}_{n, q}^{(\alpha, \beta)}(f ; x)-f(x)\right| \leq C \omega_{2}\left(f, \sqrt{\xi_{n}(x)}\right)
$$

which proves the theorem. 


\section{Competing interests}

The authors declare that they have no competing interests.

\section{Authors' contributions}

All authors contributed equally to the writing of this paper. All authors read and approved the final manuscript.

\section{Author details}

${ }^{1}$ Department of Mathematics, Indian Institute of Technology Roorkee, Roorkee, 247667, India. ${ }^{2}$ Department of Mathematics, Faculty of Science and Arts, Abant Izzet Baysal University, Bolu, 14280, Turkey.

\section{Acknowledgements}

The authors are extremely grateful to the reviewers for a careful reading of the manuscript and making valuable suggestions leading to a better presentation of the paper. The last author is thankful to the 'Council of Scientific and Industrial Research', India, for financial support to carry out the above research work.

Received: 18 June 2014 Accepted: 1 October 2014 Published: 03 Nov 2014

\section{References}

1. Lupas, A: A q-analogue of the Bernstein operator. In: Seminar on Numerical and Statistical Calculus (Cluj-Napoca, 1987), vol. 9, pp. 85-92. Univ. 'Babeş-Bolyai', Cluj-Napoca (1987)

2. Phillips, GM: Bernstein polynomials based on the $q$-integers. The heritage of P. L. Chebyshev: a Festschrift in honour of the 70th birthday of Prof. T. J. Rivlin. Ann. Numer. Math. 4(1-4), 511-518 (1997)

3. Derriennic, MM: Modified Bernstein polynomials and Jacobi polynomials in q-calculus. Rend. Circ. Mat. Palermo Suppl. 76, 269-290 (2005)

4. Gupta, V: A note on q-Baskakov-Szász operators. Lobachevskii J. Math. 31(4), 359-366 (2010)

5. Gupta, V, Heping, W: The rate of convergence of $q$-Durrmeyer operators for $0<q<1$. Math. Methods Appl. Sci. 31(16), 1946-1955 (2008)

6. Stancu, DD: Approximation of functions by a new class of linear polynomial operators. Rev. Roum. Math. Pures Appl. 13(8), 1173-1194 (1968)

7. Buyukyazici, I: On Stancu type generalization of $q$-Baskakov operators. Math. Comput. Model. 52(5-6), 752-759 (2010)

8. Agrawal, PN, Gupta, V, Sathish Kumar, A: On q-analogue of Bernstein-Schurer-Stancu operators. Appl. Math. Comput. 219(14), 7754-7764 (2013)

9. Agrawal, PN, Sathish Kumar, A, Sinha, TAK: Stancu type generalization of modified Schurer operators based on q-integers. Appl. Math. Comput. 226, 765-776 (2014)

10. Gupta, V, Karsli, H: Some approximation properties by q-Szász-Mirakyan-Baskakov-Stancu operators. Lobachevskii J. Math. 33(2), 175-182 (2012)

11. Verma, DK, Gupta, V, Agrawal, PN: Some approximation properties of Baskakov-Durrmeyer-Stancu operators. Appl. Math. Comput. 218(11), 6549-6556 (2012)

12. Gupta, V, Srivastava, GS, Sahai, A: On simultaneous approximation by Szász-beta operators. Soochow J. Math. 21(1), 1-11 (1995)

13. Gupta, V: Error estimation for mixed summation-integral type operators. J. Math. Anal. Appl. 313(2), 632-641 (2006)

14. May, CP: Saturation and inverse theorems for combinations of a class of exponential type operators. Can. J. Math. 28(6), 1224-1250 (1976)

15. Gupta, V, Noor, MA: Convergence of derivatives for certain mixed Szász-beta operators. J. Math. Anal. Appl. 321(1), 1-9 (2006)

16. Aral, A: A generalization of Szász-Mirakjan operators based on q-integers. Math. Comput. Model. 47(9-10), 1052-1062 (2008)

17. Aral, A, Gupta, V: The q-derivative and applications to q-Szász-Mirakyan operators. Calcolo 43(3), 151-170 (2006)

18. Agratini, O, Doğru, O: Weighted approximation by q-Szász-King type operators. Taiwan. J. Math. 14(4), 1283-1296 (2010)

19. Örkcü, M, Doğru, O: q-Szász-Mirakjan Kantorovich type operators preserving some test functions. Appl. Math. Lett. 24(9), 1588-1593 (2011)

20. Örkcü, M, Doğru, O: Statistical approximation of a kind of Kantorovich type q-Szász-Mirakjan operators. Nonlinear Anal. 75(5), 2874-2882 (2012)

21. Aral, A, Gupta, V, Agarwal, RP: Applications of q-Calculus in Operator Theory. Springer, Berlin (2013)

22. Kac, V, Cheung, P: Quantum Calculus. Springer, New York (2002)

23. DeVore, RA, Lorentz, GG: Constructive Approximation. Springer, Berlin (1993)

24. Ibikli, E, Gadjieva, EA: The order of approximation of some unbounded function by the sequences of positive linear operators. Turk. J. Math. 19(3), 331-337 (1995)

25. Gadjieva, AD: A problem on the convergence of a sequence of positive linear operators on unbounded sets, and theorems that are analogous to P. P. Korovkin' theorem. Dokl. Akad. Nauk SSSR 218, 1001-1004 (1974) (in Russian); Sov. Math. Dokl. 15, 1433-1436 (1974) (in English)

26. Doğru, O, Gadjieva, EA: 'Agirlikli uzaylarda Szász tipinde operatörler dizisinin sürekli fonksiyonlara yaklasimi', ll. Kizilirmak Uluslararasi Fen Bilimleri Kongresi Bildiri Kitabi, 29-37, Kirikkale (1998) (Konusmaci: O. Dogru) (Türkçe olarak sunulmus ve yayinlanmistir)

27. Lopez-Moreno, AJ: Weighted simultaneous approximation with Baskakov type operators. Acta Math. Hung. 104(1-2), 143-151 (2004)

28. Lenze, B: On Lipschitz type maximal functions and their smoothness spaces. Indag. Math. 50(1), 53-63 (1988)

29. Kolk, E: Matrix summability of statistically convergent sequences. Analysis 13(1-2), 77-83 (1993)

30. Doğru, O, Örkcü, M: Statistical approximation by a modification of q-Meyer-König and Zeller operators. Appl. Math. Lett. 23(3), 261-266 (2010)

31. Duman, O, Orhan, C: Statistical approximation by positive linear operators. Stud. Math. 161(2), 187-197 (2004) 
32. Ersan, S, Doǧru, O: Statistical approximation properties of q-Bleimann, Butzer and Hahn operators. Math. Comput. Model. 49(7-8), 1595-1606 (2009)

33. Gadjieva, AD, Orhan, C: Some approximation theorems via statistical convergence. Rocky Mt. J. Math. 32(1), 129-138 (2002)

34. Gupta, V, Radu, C: Statistical approximation properties of q-Baskakov-Kantorovich operators. Cent. Eur. J. Math. 7(4), 809-818 (2009)

35. Örkcü, M, Doğru, O: Statistical approximation of a kind of Kantorovich type $q$-Szász-Mirakjan operators. Nonlinear Anal. 75(5), 2874-2882 (2012)

36. Örkcü, M, Doğru, O: Weighted statistical approximation by Kantorovich type $q$-Szász-Mirakjan operators. Appl. Math Comput. 217(20), 7913-7919 (2011)

37. Özarslan, MA, Aktuğlu, H: A-statistical approximation of generalized Szász-Mirakjan-beta operators. Appl. Math. Lett. 24(11), 1785-1790 (2011)

38. King, JP: Positive linear operators which preserve $x^{2}$. Acta Math. Hung. 99(3), 203-208 (2003)

10.1186/1029-242X-2014-441

Cite this article as: Agrawal et al.: Szász-Baskakov type operators based on $q$-integers. Journal of Inequalities and

Applications 2014, 2014:441

Submit your manuscript to a SpringerOpen ${ }^{\odot}$ journal and benefit from:

- Convenient online submission

Rigorous peer review

- Immediate publication on acceptance

- Open access: articles freely available online

- High visibility within the field

- Retaining the copyright to your article

Submit your next manuscript at $>$ springeropen.com 